\title{
Thyroid Surgery Using New Instruments
}

\author{
HIROYUKI YAMADA, RYOJI ISHIDA, SATOSHI NAKAMURA
}

Department of Otorhinolaryngology, Yamada Red Cross Hospital, Japan

\begin{abstract}
Objective. Thyroid surgery is often conducted by two operators. It is difficult to provide the sufficient operation field by only two operators. And, we need adequate instrument for the cutting of the thyroid isthmus.

Methods. We use the fishing hooks to retract the skin flaps and strap muscles for the provision of the sufficient operation field. And, we use a vertical curved forceps to cut the isthmus for division the thyroid lobes.

Results. 646 patients were operated in our department during the period of 11 year and 10 months. For almost all patients, fishing hooks and forceps were used, and it is usefull and safe for the operations.

Conclusions. We explain our technique to carry out thyroid surgery by two operators. Fishing hook is beneficial to provide the sufficient operation field, and easy for fixation and retoutchment. Vertical curved forceps is usefull to cut the isthmus for division of the balateral lobes.
\end{abstract}

Key words: Thyroid surgery, Fishing hook, Vertical curved forceps, Surgical instruments

\section{INTRODUCTION}

Although thyroid surgery is to be conducted in the limited operation field, provision of adequate operation fields is indispensable for the safe and successful surgical treatment. In the hospitals who have many otorhinolaryngologists, operations can be carried out by many surgeons and assistants including those who fix retractors to provide sufficient operation fields. These operations, however, can not be conducted in the hospitals who have not many otorhinolaryngologists. Ideally thyroid surgery should be conducted by two surgeons because of the limited operation field, if adequate operation fields are provided. The number of surgeons available for thyroid surgery is limited at our department and this type of surgical procedure has been performed by only two surgeons since 1986. In the present study, we would like to explain the technique to carry out thyroid surgery by two surgeons including the method to provide the adequate operation field.

\section{SUBJECT}

During the period of 11 year and 10 months from July, 1986 to April, 1998, a total of 646 patients underwent thyroid surgery in the department of Otorhinolaryngology, Yamada Red Cross Hospital. These cases included differentiated thyroid cancer $(n=362)$, medullary carcinoma $(n=2)$, anaplastic carcinoma $(n=9)$, benign nodular goiter $(n=238)$, and Basedow's disease and chronic thyroiditis $(n=35)$. Of 373 patients with thyroid cancer, 42 underwent selective neck dissection of linternal jugular chain (Level V, VI, and/ or VII) on the affected side as well as the anterior compartment (Level I, II, III, and IV).

\section{THYROID OPERATION IN OUR DEPARTMENT}

In the cases of benign tumors or well-differentiated thyroid cancers which needs only dissection of anterior compartment, we make symmetric collar incisions at the site two finger breadths 
above the suprasternal notch. In the cases which require, in advance, neck dissection of jugular chain lymph nodes, the collar incision should be extended toward the head as high as the mastoid process. Another technique which can be indicated in these cases consists of MacFee skin incision.

In the present study, we will explain the thyroid surgery completed only by collar incision in our department.

\section{OPERATIVE PROCEDURES}

After the preparation of skin flaps, three fishing hooks, which are newly designed, (Fig. 1) are attached respectively to the upper and lower flaps so they can be fixed to the drape (Fig. 2). The strap muscles are divided right and left at the linea alba and the thyroid gland is dissected from the sternothyroid muscle. The fishing hooks attached to the skin flaps are transiently removed. They are set on the strap muscles and reattached to the drape (Fig. 3).

Regardless of the size to be resected by thyroidectomy, the thyroid isthmus is separated from the trachea before the thyroidectomy. This procedure, however, is not indicated in the patients with tumors in the thyroid isthmus. After the thyroid isthmus is completely separated, two pairs of vertically curved ligament forceps (SANRITU Co., Ltd.) (Fig. 4) are used to seize the isthmus so that the division of the thyroid gland can be made between the two seized sites (Fig. 5). The stumps are ligated for hemostasis.

The procedure taken afterward is nearly the same as the routine surgical technique adopted at many hospitals. The thyroid gland is grasped and pulled to confirm the recurrent laryngeal nerve at the level of thyroid lower pole. The inferior thyroid vein and artery are ligated and dissected and the recurrent nerve is identified at the caudal side. The thyroid gland is dissected while the recurrent laryngeal nerve is well preserved. In cases the ligation of the vessels of the thyroid upper pole is necessary, the assistant used hook retractors to provide a sufficient operation field, because adequate operation fields can not be provided by using the abovementioned fishing hooks. The superior thyroid vein and artery are ligated and dissected. During this procedure, care must be taken not to damage the external branch of the superior laryngeal nerve.

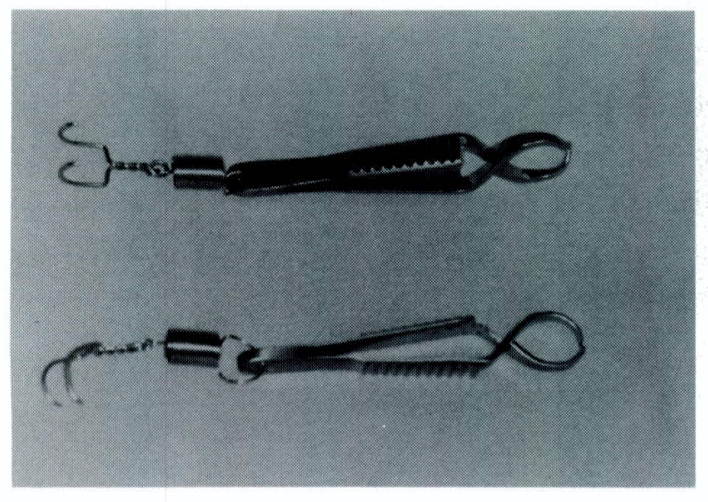

Fig. 1 Fishing hooks are connected to the spring and forceps that graps the drape.

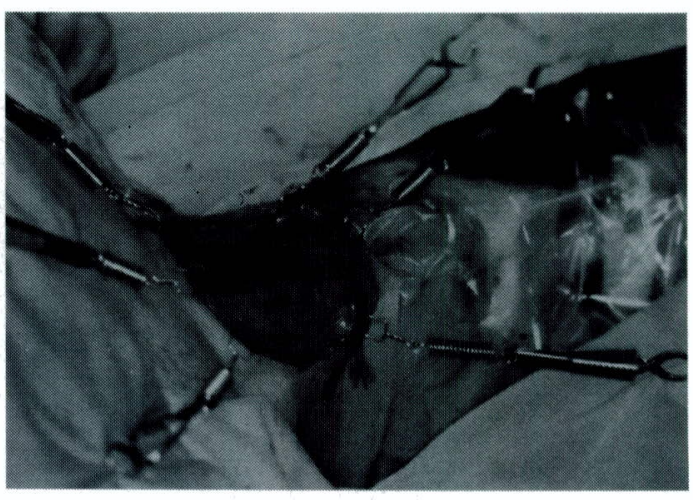

Fig. 2 Cervical skin flap is retracted by 6 fishing hooks. 


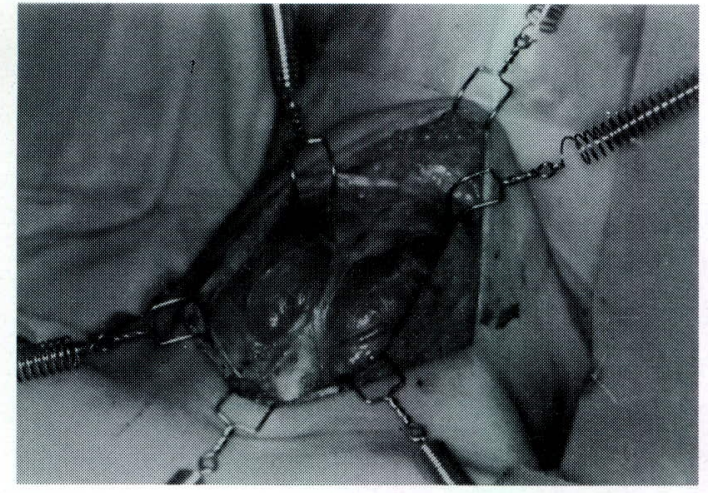

Fig. 3 Strap muscles are retracted by 4 fishing hooks and the thyroid gland is exposed.

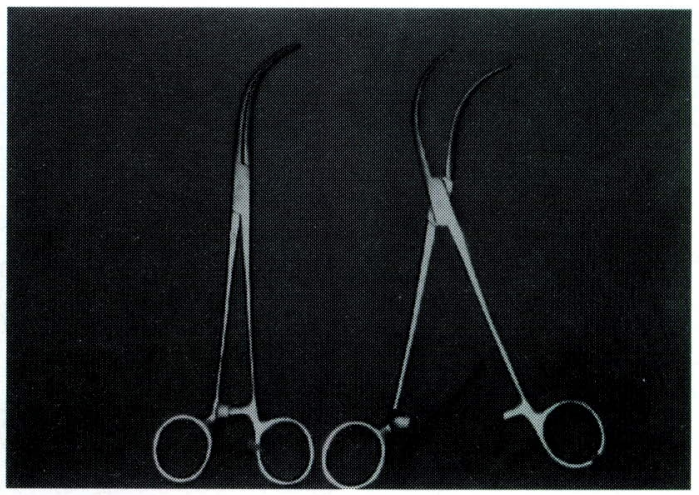

Fig. 4 Vertically curved ligament forceps.

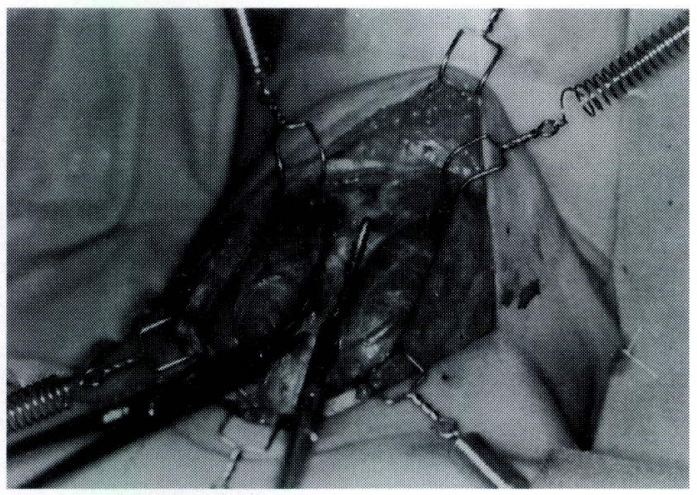

Fig. 5 Thyroid isthmus is seized by 2 ligament forcepses.

Berry's ligament is dissected and thyroidectomy is completed. In differentiated thyroid cancer cases, the additional excision of anterior compartment is to be conducted. If possible, the parathyroid gland should be preserved on the spot along with the branches of the superior thyroid veins and arteries.

A drain is placed at the site where the thyroid gland is excised and the right and left strap muscles are stiched. The platysma and the skin are approximated by nylon sutures.

\section{DISCUSSION}

At this hospital we often operate on the patients with thyroid neoplasms of smaller diameter. Because of the limited number of available staffs and the limited operation field of thyroid surgery, only two surgeons usually participate in this surgery. In order to conduct safe and effective thyroid surgery, we adopt the following technique.

A skin flap is prepared by making a collar incision of the lower part of neck. How to fix this skin flap should be carefully considered because providing the adequate operation fields and skillful manipulations depends on the effective fixation. If the thyroid surgery is conducted by 
more than three surgeons, fixation the skin flap presents no serious problem because the second assistant fixes the retractor. Therefore, this technical problem arises only in the operations to be carried out by two surgeons.

In these operations, an assistant fixes a retractor to secure the adequate operation field ${ }^{1,2)}$. During the ligation of vessels, however, the assistant has to hand transiently the retractor to the operator. This indicates the waste of time and the risk of technical complication.

Sufficient operation fields can be obtained by fixing the skin flap with needles and nylons ${ }^{3,4)}$. This technique enables the operators to use their both hands for effective manipulation. However, the trouble is that the fixation of the skin flap results in the formation of the additional wound in the neck. In case of the change of the fixation, the intricate manipulation and the prolongation of treatment are necessary.

At some hospitals, wound retractors such as those used in thoracic or abdominal surgery are developed and used in thyroid surgery. These devices, however, are not useful because of their disadvantages such as complicated manipulation for fixation and removal and narrowed operation fields. Compared with other surgical procedures, the use of these retractors seems to be less cost-effective and disadvantageous.

The fishing hook we use for thyroid surgery is a hook developed by slightly modifying the hook used in neurosurgery. The tip of the fishing hook which is to be attached to the skin flap is slightly rounded to minimize the tissue damage. The spring which is connected with the hook enables the user to pull the skin flap with the optimum force. In order to ensure the fixation to the drape, a pair of forceps is also connected with the hook. The upper and lower skin flaps are respectively fixed with the three hooks so that the adequate operation fields can be obtained. When the strap muscles are divided right and left, the hook used for the fixation of the skin flap can be transiently removed so that it can be placed on the strap muscles. This technique enables the operator to safely complete various procedures other than the treatment of the thyroid upper pole. In the cases of great struma and Basedow's disease and chronic thyroiditis accompanied by thyromegaly, sufficient operation fields can be ensured by making a transverse incision of strap muscles.

Care must be taken not to attach the fishing hook to the soft tissues with important vessels and nerves. Fortunately we recognized no tissue damage due to the attachment of the hook. This fishing hook can be used not only in thyroid surgery but also in the operations for tumors of the head and neck region. Compared with other methods to provide operation fields, the use of fishing hook is more useful because of its maneuvability and noninvasiveness.

The problem of this hook is weakness of retraction of the soft tissures due to the small hook size. Furthermore, if we would like to not make the incision of strap muscles in cases of large tumors, more strong retraction of the strap muscles using the usual retractors is necessary.

We adopt the method to divide the thyroid gland into two portions at its isthmus regardless of the size of thyroid gland to be resected. This technique enables us to control the amount of bleeding during thyroidectomy and to grasp and pull the portion of thyroid gland to be resected without difficulty.

Generally Kelly's forceps, Pean's forceps and Kocher's forceps are used for the division at thyroid isthmus. The insertion of two of these forceps into the latent space between the isthmus and trachea requires sophisticated skills. The vertically curved forceps we use in thyroid surgery 
can be inserted into the space with relatively ease because the tips of the forceps are curved and can be opened in the curved direction. This technique will give to the surgions reduction of operation time and unnecessary bleeding from the thyroid isthmus.

It is necessary for operators to make sufficient retraction of the skin flap and the strap muscles in thyroid surgery because of the limited operation fields. In the present study we explained how to complete thyroid surgery safely and effectively only by two operators. This operation technique has enabled us to conduct safe thyroid surgery without causing any accidental tissue damage.

\section{REFERENCES}

1) Esaki, H: Surgical technique of Basedow's disease. Surgery of thyroid (ed 2). Japan, Health Pub, 1987, pp 28-58.

2) Kasai, N : Surgical procedures of thyroid and anterior neck. Technique of thyroid resection and management of patients (ed 1). Japan, Igaku Shoin, 1987, pp 53-74.

3) Fujimoto, Y : Surgery of Basedow's disease. Surgical MOOK, vol 27. Japan, Kanehara Pub, 1982, pp 137-143.

4) Matsuura, H: Standard operation for well-differenciated thyroid cancer. Surgical MOOK, vol 27. Japan, Kanehara Pub, 1982, pp 189-210. 